\title{
Prácticas de enseñanza de profesores en contextos interculturales: obstáculos y desafíos
}

Teaching Practices of Teachers in Cross-Cultural Contexts: Obstacles and Challenges

Fecha de recepción: 17 DE MAYO DE 2016 / Fecha de aceptación: 6 DE MARZO DE 2018 / Fecha de disponibilidad en línea: ENERO DE 2019

(c) (1)

\section{(1)}

doi: 10.11144/Javeriana.m11-23.pepc

Juan Carlos Beltrán-Véliz jbeltranucatolica@gmail.com Universidad Mayor de Temuco, Chile https://orcid.org/0000-0003-4614-0650

Juan Guillermo Mansilla-Sepúlveda jmansilla@uct.cl

Universidad Católica de Temuco, Chille https://orcid.org/0000-0001-8175-7475
Carlos Felimer del Valle-Rojas carlos.delvalle@ufrontera.cl Universidad de la Frontera de Temuco, Chile https://orcid.org/0000-0002-9905-672X

Braulio Ademir Navarro-Aburto braulio.navarrro@uautonoma.cl Universidad de la Frontera de Temuco, Chile https://orcid.org/0000-0002-3676-6222

\section{Resumen}

Se propuso el objetivo: develar analíticamente los factores que obstaculizan y facilitan las prácticas de enseñanza de profesores interculturales y tradicionales ${ }^{1}$ en escuelas situadas en contextos interculturales en la precordillera de la Araucanía. Se planteó un diseño cualitativo. Los resultados develan prácticas de enseñanza focalizadas en las clases expositivas. Asimismo, se evidencia ausencia de articulación pedagógica y de prácticas reflexivas, lo cual dificulta los procesos de enseñanza-aprendizaje.

\section{Palabras clave}

Educación intercultural; cultura; método de enseñanza; conocimientos indígenas

\section{Abstract}

The proposed objective consisted in exposing analytically the factors hindering and those making easier the learning practices of both cross-cultural and traditional teachers ${ }^{2}$ in schools situated in cross-cultural contexts in the foothills of Araucanía. To do so a qualitative design was set out. The results showed learning practices focused on the lecture-like classes. Likewise, it is evident a lack of pedagogical articulation and reflection practices, which makes difficult the teaching-learning processes.

\section{Keywords}

Intercultural education; culture; teaching methods; local knowledge

Para citar este artículo / To cite this article

Beltrán-Véliz, J. C.; Masilla-Sepúlveda, J. G.; Del Valle-Rojas, C. B. del \& Navarro-Aburto, B. A. (2019). Prácticas de enseñanza de profesores en contextos interculturales: obstáculos y desafíos. magis, Revista Internacional de Investigación en Educación, 11 (23), 5-22. doi: 10.11144/Javeriana.m11-23.pepc

1 En el texto, este concepto se refiere a los profesores no mapuche; vale decir, a profesores con una formación denominada "occidentalizada", debido a que el proceso de enseñanza aprendizaje se orienta preferentemente desde el currículo oficial.

2 The concept refers herein to non-Mapuche teachers. It is worth clarifying that it refers to teachers educated under the so-called "westernized" education because their teaching-learning process is preferentially guided by the official curriculum. 


\section{Introducción}

Uno de los problemas que se visualizan en Chile se relaciona con la equidad y calidad de la educación expresada en la falta de procesos, y resultados que se traduzcan en buenas prácticas pedagógicas (Beltrán Véliz, 2014). Esto con la finalidad de que los profesores contribuyan a generar en los estudiantes aprendizajes de calidad. La Organización para la Cooperación y el Desarrollo Económicos indica que la calidad de los profesores y de su enseñanza es el factor más importante para explicar los resultados de los alumnos (OCDE, 2005). Al respecto, "Existe un consenso emergente acerca de que el profesorado influye de manera significativa en el aprendizaje de los alumnos y en la eficacia de la escuela" (Cochran-Smith \& Fries, 2005, p. 40).

En este contexto, la enseñanza del conocimiento entre la cultura mapuche y la occidental es un proceso complejo, pues el docente, quien posee el conocimiento, entrega los contenidos al alumnado acorde a sus habilidades y capacidades pedagógicas. En cuanto a este punto, Juan Carlos Beltrán Véliz y Sonia Eliana Osses Bustingorry (2018, pp. 671-672) evidencian que: "la educación que se entrega en contextos indígenas e interculturales, y específicamente en el caso de contextos mapuche, se conservan prácticas educativas tradicionales enmarcadas en el conocimiento occidental $y$, por tanto, descontextualizadas. En tal sentido, se observa falta de dominio disciplinar respecto de métodos de enseñanza indígenas por parte de los profesores y profesoras". En relación con tal hecho, se hace necesario que los docentes contextualicen sus prácticas pedagógicas y que dispongan de un conocimiento adecuado de cómo enseñar. En esta línea, Segundo Quintriqueo (2010) subraya que la educación escolar carece de una relación educativa con el saber y conocimiento mapuche para la formación de niños y adolescentes. En este contexto, en un estudio realizado en Ch'ol Chiapas por José Bastiani Gómez, Lorena Ruiz-Montoya, Erín Estrada Lugo, Tania Cruz Salazar y José Antonio Aparicio Quintanilla (2012) indican que durante la observación del trabajo en el aula se constató que este se da en un ambiente de autoridad vertical, pues el profesor decide las actividades que se realizarán sin mayor consideración de las capacidades y habilidades de los estudiantes. Tampoco se observó que los profesores propiciaran ambientes de trabajo nuevos y de mayor armonía.

Por su parte, Gloria Quidel Catrilaf (2011) se refiere a las fallas en las estrategias empleadas para enseñar la lengua mapuche (mapudungun, habla de la tierra) y destaca que persisten prácticas como la improvisación, el uso de la lista de vocabulario y la ausencia de producción en la lengua. En relación con la afirmación anterior, los docentes deben incorporar en sus prácticas de enseñanza no solamente el dominio de contenido disciplinario puro, sino que además les exige competencias asociadas a la apropiación del conocimiento didáctico para vehiculizar el saber disciplinario hacia el saber pedagógico, con el objeto de cumplir con el acto educativo (Pellón Arcaya, Mansilla Sepúlveda \& San Martín Cantero, 2009). En esta lógica, se espera que el profesor posea un manejo acabado del conocimiento didáctico del contenido, teniendo en cuenta el contexto y las características de los estudiantes. En concordancia con lo anterior, Segundo Quintriqueo, Héctor Torres, Maritza Gutiérrez y Dykssa Sáez (2011) señalan que "Ios estudiantes aprenden en la diferencia, considerando el conocimiento indígena y no indígena como una riqueza de la producción humana, para mejorar la calidad de los aprendizajes, las oportunidades y la convivencia social". En este sentido, las prácticas de enseñanza deben orientarse hacia un propósito común con base en los requerimientos de 
la sociedad actual como el aprendizaje en equipo que integre a todos a partir de su diversidad y, desarrollar conciencia de la complejidad de la tarea de educar (Wertsch, 1991). En tal sentido, se debe producir una transformación de saberes sabios, lo cual implica cambios paradigmáticos; sobre este particular, Thomas Kuhn (1962) señala que debe ocurrir una verdadera revolución científica y provocar una crisis que tensiona el paradigma dominante y da paso al surgimiento de un nuevo paradigma con núcleos teóricos emergentes para constituirse en ciencia normal. En este sentido, se debe reflexionar en torno a las prácticas pedagógicas. Al respecto, Donald Schön (1992) plantea:

...una nueva epistemología de la práctica que pusiera de relieve la cuestión del conocimiento profesional asumiendo como punto de partida la competencia y el arte que forman parte de la práctica efectiva; sobre todo, la reflexión en acción ('el pensar en lo que se hace mientras se está haciendo') (p. 9).

En esta lógica, surge la propuesta de transposición didáctica, la cual según Yves Chevallard (1991) permite la transformación del contenido para su enseñanza, es decir, la transposición didáctica del contenido. El profesor, entendido como aquel que construye un conocimiento acerca de la disciplina, busca los sentidos, formas y medios para enseñar aquel contenido que pretende construir con sus alumnos; asimismo, posee diversos conocimientos (Shulman, 2004) que están presentes en todo momento en su quehacer profesional (Mansilla Sepúlveda \& Beltrán Véliz, 2013). Desde esta perspectiva, la transposición didáctica cobra relevancia puesto que se requiere que un docente posea conocimiento amplio y profundo de cómo y cuándo debe enseñar, con una conducción apropiada de estrategias de enseñanza y aprendizaje, recursos didácticos e instrumentos de evaluación, para lo cual se debe tener en cuenta el contexto. Por lo tanto, la transposición didáctica de profesores que actúan en contextos interculturales supone un cambio en el proceso enseñanza-aprendizaje. A la luz de los antecedentes, surge la siguiente pregunta:

¿Cuáles son los factores que obstaculizan y facilitan las prácticas de enseñanza de profesores interculturales y tradicionales en las escuelas situadas en contextos interculturales en la precordillera de la Araucanía?

El objetivo del presente trabajo es develar analíticamente los factores que obstaculizan y facilitan las prácticas de enseñanza de profesores interculturales y tradicionales en escuelas situadas en contextos interculturales en la precordillera de la Araucanía.

\section{Educación intercultural}

María Teresa Aguado Odina (1991) sostiene que la educación intercultural alude a la tendencia reformadora en la práctica educativa, y variada en sus metas, con la que se intenta responder a la diversidad provocada por la confrontación y convivencia de diferentes grupos étnicos y culturales en el seno de una sociedad dada. Actualmente se habla de educación intercultural como una propuesta de acción educativa teórico-práctica en la que prevalece el reconocimiento de la existencia de los "otros" como sujetos poseedores de una cultura diferente y el conocimiento de lo que esto significa en términos de semejanzas y diferencias con la propia cultura escolar caracterizada por múltiples influencias (Díaz-Aguado, 2002). La educación intercultural "se caracteriza por el intercambio y la interacción y favorece el desarrollo personal humano, para lo cual debemos adquirir unas competencias y unas habilidades interculturales" (Sáez Alonso, 2006, p. 1). En tal sentido, "la educación intercultural debe buscar promover en los estudiantes el desarrollo de capacidades de pensamiento crítico, que les permita generar una implicancia entre sujetos en la necesidad de construir un proyecto social en común" (Quintriqueo, Morales, Quilaqueo \& Arias, 2016, p. 13).

En este plano, la educación intercultural en sí solo tendrá significación, impacto y valor cuando esté asumida de manera crítica (Walsh, 2009). Esto permite que la educación intercultural se construya desde una valoración compartida que responde al desafío de respetar las diferencias sociales, culturales e individuales (Quintriqueo, Morales, Quilaqueo \& Arias, 2016).

\section{El rol del profesor en la educación intercultural}

En este campo, José Antonio Jordán (1999, p. 65) afirma que "la figura del profesor se convierte en el instrumento pedagógico por excelencia". Al respecto, Javier Serrano Ruiz (1998) plantea que el maestro debe ser un "abridor de mundo", desde sus propias raíces y para la comprensión de la situación de sus alumnos. En este plano, Segundo Quintriqueo y Margaret McGinity Travers (2009) sostienen que el desarrollo de prácticas pedagógicas debe orientarse en el diálogo de saberes sustentadas en: (i) la mediación del profesor/a en el aprendizaje y comunicación intercultural de alumnos/as de ascendencia mapuche y no mapuche, (ii) una pedagogía que considere la relación del saber mapuche, profesional y disciplinario (escolar) en la formación de personas. Entonces, el profesor es el agente clave de la educación intercultural. En tal sentido, el rol docente ha de estar focalizado en 
implementar prácticas pedagógicas contextualizadas basadas en estrategias de enseñanza y aprendizaje que, con diálogo, reflexión y creatividad, promuevan el desarrollo del pensamiento crítico y la autonomía del estudiante. En este contexto, Stefano Claudio Sartorello (2014) señala que en particular, se conjuntaron dos aspectos relevantes sobre los cuales nuestros colaboradores pusieron énfasis a lo largo de todo el proceso de elaboración del perfil del maestro y que enfatizan principalmente la dimensión del ser del maestro. En cuanto acompañante, el maestro no dirige o conduce el proceso de enseñanza-aprendizaje, sino que ayuda a los niños en su proceso de desarrollo que empieza desde los conocimientos, habilidades y valores que adquieren en sus casas y en el territorio de la comunidad. El acompañante reconoce a los niños como sujetos activos y autónomos del aprendizaje y su papel radica entonces en acompañarlos en un proceso que no depende únicamente de él, sino en el cual estos desempeñan un papel protagónico.

\section{Transposición didáctica}

En este contexto, surge la idea del Conocimiento Didáctico del Contenido (en adelante, CDC). Al respecto, José Antonio Acevedo Díaz (2009) indica que el origen del CDC se remonta a la conferencia El paradigma perdido en la investigación sobre la enseñanza, que Lee Shulman dictó en la Universidad de Texas, en Austin durante el verano de 1983 (Shulman, 1999). Este paradigma resultó ser el pensamiento del profesor sobre el contenido del tema objeto de estudio y su interacción con la didáctica (Berry, Loughran \& Van Driel, 2008; Garritz, 2006; Garritz \& Trinidad-Velasco, 2004).

Según Lee Shulman (2005), la base de conocimientos del profesor — como mínimo - incluiría las categorías: conocimiento del contenido; conocimiento didáctico general, teniendo en cuenta especialmente aquellos principios y estrategias generales de manejo y organización de la clase que trascienden el ámbito de la asignatura; conocimiento del currículo, con un especial dominio de los materiales y los programas que sirven como "herramientas para el oficio" del docente; conocimiento didáctico del contenido: esa especial amalgama entre materia y pedagogía que constituye una esfera exclusiva de los maestros, su propia forma especial de comprensión profesional; conocimiento de los alumnos y de sus características; conocimiento de los contextos educativos, que abarcan desde el funcionamiento del grupo o de la clase, la gestión y financiación de los distritos escolares, hasta el carácter de las comunidades y culturas; y el conocimiento de los objetivos, las finalidades y los valores educativos, y de sus fundamentos filosóficos e históricos.
Entre estas categorías, el conocimiento didáctico del contenido adquiere particular interés porque identifica los cuerpos de conocimientos distintivos para la enseñanza. Representa la mezcla entre materia y didáctica por la que se llega a una comprensión de cómo determinados temas y problemas se organizan, se representan y se adaptan a los diversos intereses y capacidades de los alumnos, y se exponen para su enseñanza (Shulman, 2005). Esto implica que el profesor debe transformar, contextualizar y organizar los saberes para trasladarlos a la enseñanza, a fin de que los estudiantes puedan comprenderlos.

Además, es preciso señalar que una de las más relevantes reformulaciones del modelo teórico de Shulman es el planteamiento de Elizabeth Fennema y Megan Loef Franke (1992, citadas por Alicia Zamorano Vargas, 2015), que proponen un modelo basado en el paradigma de la enseñanza de Shulman que incluye la idea de que los conocimientos necesarios para la enseñanza son interactivos y dinámicos. Este modelo además agrega el conocimiento del pensamiento de los alumnos. Actualmente, estos planteamientos se han complejizado a partir del modelo teórico didáctico elaborado por Tim Rowland y Fay Turner (2007) en la Universidad de Cambridge, el Knowledge Quartet, también denominado "cuarteto del conocimiento" que integra cuatro dimensiones: fundación, transformación, conexión y contingencia.

En relación con lo expuesto, desde el campo de interculturalidad, el CDC cobra relevancia, dado que Shulman (2005) señala que los profesores deben desarrollar un conocimiento específico de cómo enseñar su materia específica, por lo que se requiere un CDC, propio del buen hacer docente. Al respecto, José Antonio Acevedo Díaz (2009) dice que el CDC facilita la comprensión de cómo un profesor que "conoce una materia" se convierte poco a poco en "maestro de la materia" (Clermont, Borko \& Krajcik, 1994; Marcelo, 2001; Mulholland \& Wallace, 2005), mediante la utilización de una serie de recursos y estrategias didácticas (Mansilla Sepúlveda \& Beltrán Véliz, 2013). En este contexto, Yves Chevallard (1991) maneja un concepto similar al del CDC, el de transposición didáctica (Mansilla Sepúlveda \& Beltrán Véliz, 2013). Chevallard (1991) la define como:

Un contenido de saber que ha sido designado como un saber para enseñar, sufre un conjunto de transformaciones adaptativas que van a hacerlo apto para ocupar un lugar entre los objetos de enseñanza. El "trabajo" que transforma un objeto de saber a enseñar en un objeto de enseñanza, es denominado la transposición didáctica (p. 45). 
Es decir, la responsabilidad del profesor es transformar el saber sabio (o sea, el saber científico o artístico) en un saber enseñado, con el fin de que los estudiantes puedan comprender ese lenguaje y puedan apropiarse de este conocimiento (Grisales-Franco \& González-Agudelo 2009). Desde este plano, es preciso abordar el modelo integrador del CDC de Julie GessNewsome (1999) que considera tipos de conocimiento que se constituyen en conceptos claves para yuxtaponer los saberes disciplinarios, didácticos y contextuales, complejizando la actuación docente, donde hay un desafío a la superación de una enseñanza tradicional magistocéntrica, de tal forma que esta se oriente a una lógica paidocéntrica (Pellón Arcaya, Mansilla Sepúlveda \& San Martín Cantero, 2009). Esto se presenta en la figura 1.

Figura 1

Modelo integrador del CDC

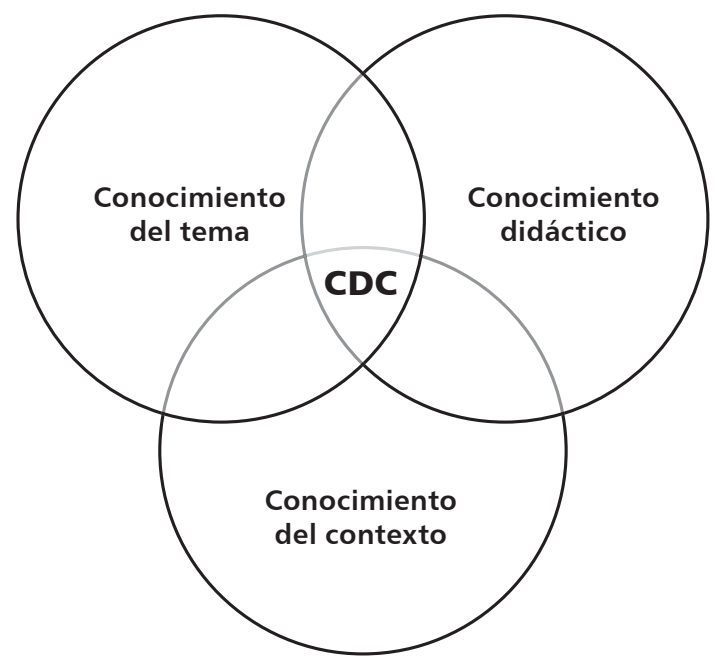

Fuente: Julie Gess-Newsome (1999, citada en Acevedo Díaz, 2009, p. 22)

En la figura 1, se aprecian interacciones que permiten la transposición didáctica contextualizada. En el contexto intercultural, Segundo Quintriqueo, Héctor Torres, Maritza Gutiérrez y Dykssa Sáez (2011, pp. 485-486) expresan que para poder articularlas, ambas lógicas de conocimiento (mapuche y no mapuche) se deben plasmar en recursos interculturales utilizados en la planificación de la acción didáctica, con base en estrategias de transposición didáctica que generan la relación dialógica de saberes educativos. Lo anterior demanda una actuación crítica y reflexiva del profesor sobre sus prácticas, y un proceso de sistematización de saberes y conocimientos educativos mapuches.

\section{Metodología}

El método de investigación es cualitativo descriptivo y hermenéutico puesto que tiene relevancia específica para el estudio de las relaciones sociales (Flick, 2007). Se usó el estudio de casos, caracterizado por ser "particularista, descriptivo y heurístico y se basa en el razonamiento inductivo al manejar múltiples fuentes de datos" (Pérez Serrano, 2001, p. 85). 
Se utilizó la teoría fundamentada (Strauss \& Corbin, 2002), para abordar el proceso de análisis mediante la construcción de categorías conceptuales, y el método comparativo constante (Mansilla Sepúlveda \& Beltrán-Véliz, 2013), dado que "los intérpretes cuidan de comparar los códigos una y otra vez con los códigos de las clasificaciones obtenidas" (Flick, 2007, p. 248). El caso está constituido por 18 profesores interculturales y 18 profesores tradicionales. La selección de los sujetos fue intencional, según Alicia Gurdián-Fernández (2007, p. 247), "la selección debe ser apropiada a las y los sujetos actuantes. Es decir, seleccionar a quienes tengan un mejor conocimiento del fenómeno por investigar. Esto garantiza una saturación efectiva y eficiente de las categorías con información de óptima calidad". Las técnicas de recolección de información fueron entrevistas semiestructuradas y observaciones etnográficas. Respecto de la primera técnica, José Ignacio Ruiz Olabuénaga (2012) señala que la entrevista semiestructurada permite al investigador dar la oportunidad de que el entrevistado pueda expresarse en sus propios términos y con la suficiente profundidad para captar toda la riqueza de su significado. En cuanto a la observación etnográfica, según Uwe Flick (2007), nace del deseo por parte del investigador de ir más allá de la palabra hablada y del informe sobre las acciones, a favor de analizar estas mismas a medida que se producen de modo natural.

En la etapa de "saturación teórica" se desarrolló el muestreo teórico donde los datos se tornaron repetitivos. Flick define el criterio de 'saturación teórica' de la siguiente manera: "(...) el muestreo e integración de material nuevo se acaba cuando la 'saturación teórica' de una categoría o grupo de casos se ha alcanzado, es decir, cuando no emerge ya nada nuevo" (Flick, 2007, p. 79).

Análisis de datos

La información se transcribió en documentos de formato Word, para realizar el análisis de los datos con el programa Atlas.ti 7.0 que, de acuerdo con Juan Muñoz Justicia (2005), es una herramienta informática cuyo objetivo es analizar principalmente grandes volúmenes de datos textuales. En una primera fase, los datos fueron reducidos y analizados en el proceso de codificación abierta (Mansilla Sepúlveda \& Beltrán Véliz, 2013). Respecto a lo anterior, Anselm Strauss y Juliet Corbin (2002, p. 134) señalan que "para descubrir y desarrollar los conceptos debemos abrir el texto y exponer los pensamientos, ideas y significados contenidos en él". En una segunda fase, se dio paso a la codificación axial; las categorías axiales se enriquecen por su ajuste con el mayor número de pasajes posibles, se relacionan las categorías y sus subcategorías (Flick, 2007). Este proceso de reducción de datos se realizó desde el programa Atlas.ti 7.0, lo que permitió la deconstrucción de las unidades hermenéuticas generadas desde las transcripciones, el levantamiento de códigos desde los documentos primarios y la generación de familias de códigos, los cuales se vincularon con la categoría central; y la generación de redes conceptuales. Posteriormente, se presentaron los resultados por códigos, y finalmente analizaron los datos por cada código.

\section{Resultados}

Desde la codificación abierta, emerge la categoría facilitadores y obstaculizadores de la enseñanza, a partir de la cual se vinculan ocho códigos: 1) diseño de la enseñanza, 2) metodología de la enseñanza, 3) fuentes de conocimiento, 4) práctica evaluativa, 5) dominio del conocimiento disciplinar y didáctico, 6) articulación pedagógica ,7) práctica reflexiva, y 8) prejuicios. Estos códigos se visualizan en la siguiente red conceptual. 
Figura 2

Factores y obstaculizadores de la enseñanza

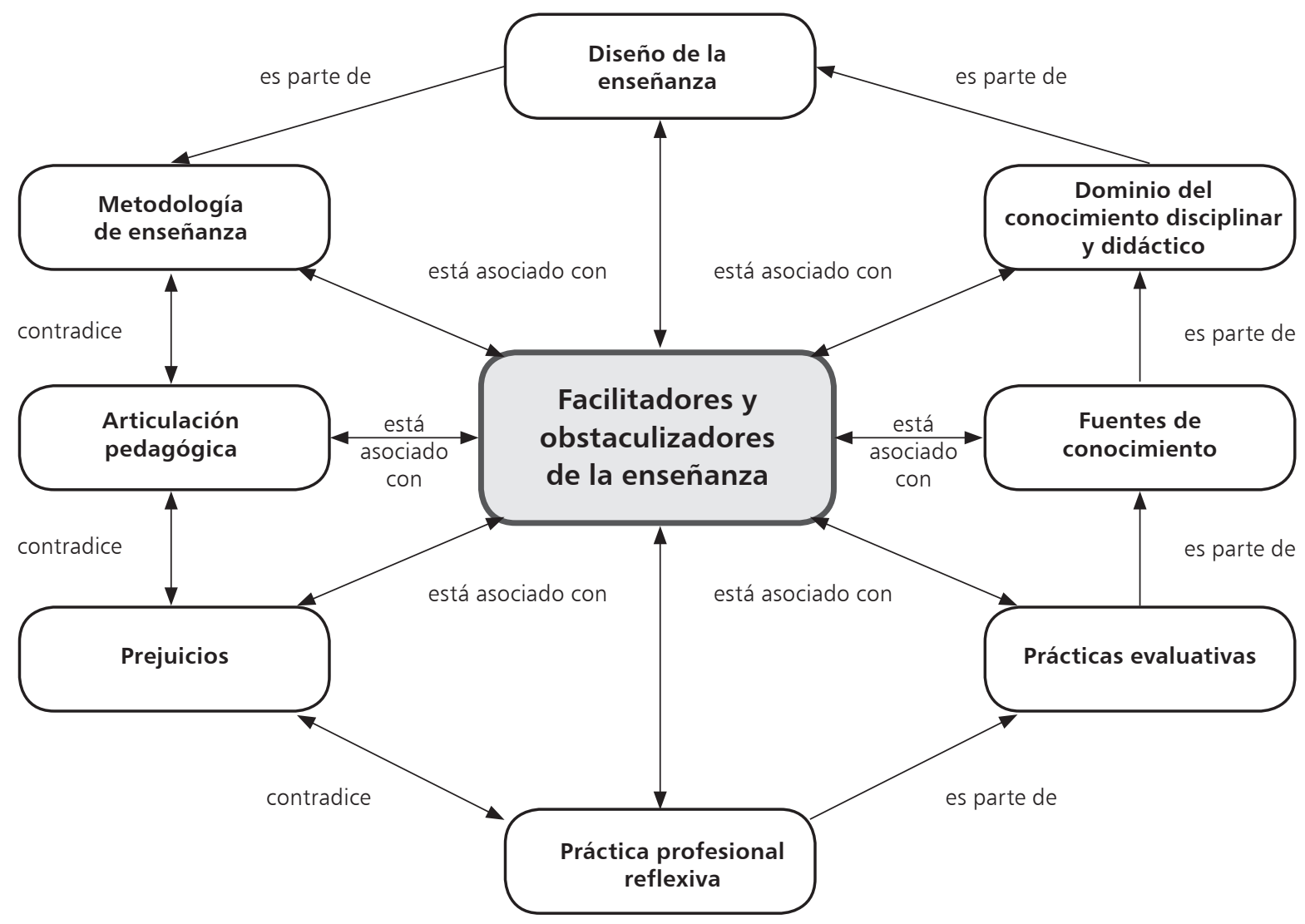

Fuente: elaboración propia

Código 1. Diseño de la enseñanza

Este código hace referencia a la forma en que los profesores tradicio-

nales e interculturales diseñan la enseñanza.

Los hallazgos dan cuenta de diseños de la enseñanza rígidos centrados en la clase expositiva y evidencian escasos espacios para la reflexión. Esto se manifiesta en las siguientes narraciones realizadas por un profesor tradicional: "...cuando tú planificas, sobre todo en las clases teóricas donde hay que pasar determinados contenidos (...) a lo más puedes hablar más, pero de esos contenidos y puntos no te puedes salir..." (E5:P8). Un profesor intercultural declara: "Debes apegarte a los contenidos, a la estructura (inicio, desarrollo y cierre) a la cual le debes establecer un tiempo específico, es decir, debes ser bien estructurado" (E7:P18). Esto implica transformaciones en el diseño de la enseñanza, dado que los profesores deben ser capaces de buscar los medios y mecanismos para producir conocimiento tomando como base las teorías y los problemas que subyacen en las culturas y en la sociedad (Giroux, 1997).

Código 2. Metodología de la enseñanza

Este código se refiere a las estrategias de enseñanza y aprendizaje que los profesores tradicionales e interculturales emplean en el desarrollo de sus clases. 
En este contexto, se evidencia que tanto los profesores interculturales como los tradicionales emplean la clase expositiva. Cabe señalar, que se presenta con mayor frecuencia en profesores tradicionales. Lo anterior se visualiza en los siguientes relatos: "Alumnos... miren el Power Point y copien en sus cuadernos los números y los objetos de la sala de clase en mapudungun" (lengua mapuche) (R2/OB2:P2). Asimismo, lo anterior se observa en la planificación de un profesor intercultural: "Se realizará una clase expositiva en la que se describe las características del pueblo mapuche: sistema de vida, política, económica, etc." (R3/OB5:P3). Respecto de tales evidencias, María Elena Mellado Hernández y Juan Carlos Chaucono Catrinao (2016) en estudio realizado en la comuna de Vilcún en la precordillera, concluyen que en el aula solo se observan prácticas de enseñanza tradicionales centradas en el currículo prescrito.

Por otra parte, es posible observar que algunos docentes tradicionales emplean con menor frecuencia la narración de cuentos, leyendas, lectura de textos, trabajo en grupo, las cuales facilitan el desarrollo de aprendizajes. Al respecto, un docente tradicional manifiesta: "Para abordar un contenido al principio o durante el desarrollo de la clase, les narro historias del pueblo mapuche, con el fin de que ellos puedan después crear sus propias historias" (E9:P3). Otro profesor declara: "Los acomodo en grupos y les paso cuentos y luego les doy algunas interrogantes para que respondan sobre lo que leyeron" (E12:22). En esta lógica, determinados profesores interculturales declaran emplear estrategias didácticas contextualizadas tanto mapuches como occidentales, pero con una menor frecuencia, como son: nütram (discurso oral), ngülam (consejo), epew (cuentos o relatos), feyentun (leyendas), piam (mitos), pewmas (sueños), trabajo en grupo y conocimientos previos. Lo señalado se evidencia en el siguiente relato: "para empezar la clase, uso conocimientos previos, además, los saberes y valores los enseño a través de la oralidad usando el nütram, ngülam, epew, feyentun, piam, pewmas y el trabajo en grupo para que trabaje colaborativamente" (E14:P4). Estas estrategias facilitan la enseñanza contextualizada, por lo cual es preciso que los profesores repliquen estrategias metodológicas con un enfoque intercultural para dar sentido al proceso de enseñanza (Quintriqueo, 2010).

\section{Código 3. Fuentes de conocimiento}

El código da cuenta de las fuentes de conocimientos a las cuales recurren generalmente los profesores tradicionales e interculturales en el desarrollo de sus prácticas de enseñanza.

Los docentes interculturales y tradicionales obtienen la información de las fuentes de conocimiento, desde su preparación profesional adquirida en su formación universitaria, información recabada en congresos, en publicaciones de investigaciones. Esto se evidencia en la siguiente declaración que emana de un profesor tradicional: "Yo busco artículos relacionados con investigaciones educativas que han sido probadas, y libros" (E10:P11). En este campo, un profesor intercultural declara: "Mi preparación como profesor intercultural me ha ayudado mucho en el contexto donde trabajo. Además, yo voy a congresos interculturales, lo cual me ha dado más preparación" (E11:P17). Por otra parte, tanto profesores tradicionales como interculturales hacen un uso excesivo de textos escolares, lo que se refleja en la siguiente evidencia obtenida de una clase de un profesor tradicional: "Bueno, alumnos, vamos a empezar la clase; saquen el texto, porque vamos a trabajar en la página 25, pero antes van a copiar las siguientes preguntas de la pizarra para que trabajen en la página que ya se les mencionó" (R4/OB7:P8). Asimismo, un profesor 
intercultural declara: "Les doy una lectura en el libro de mapudungun y luego la traducen con la ayuda de un diccionario de mapudungun, de esa manera internalizan vocabulario" (E29:P7). Por otra parte, los profesores interculturales recurren a consultar expertos: “Lo que utilizo es la conversación con la gente que tenga conocimiento, lo que en la cultura mapuche se denomina kimche (persona con conocimientos y sabia), es a quien yo le puedo hacer consultas, pues él tiene mucha experiencia" (E2:P6).

En este plano, Lee Shulman (2005) señala que hay por lo menos cuatro fuentes de conocimientos que están en la base de la enseñanza: (a) formación académica en la disciplina a enseñar, (b) materiales y contexto del proceso educativo institucionalizado, (c) la investigación sobre escolarización, aprendizaje humano y desarrollo del fenómeno sociocultural del quehacer de los profesores, y (d) la sabiduría que otorga la práctica misma.

\section{Código 4. Prácticas evaluativas}

Este código alude a los tipos de evaluación que emplean los profesores tradicionales e interculturales en el desarrollo de sus clases.

Un gran número de profesores no valora la evaluación diagnóstica, asimismo, en su mayoría, los profesores emplean la evaluación sumativa, mientras un número reducido utiliza la evaluación de proceso. Lo expresado queda manifiesto en el siguiente relato de un profesor tradicional: "La evaluación diagnóstica la mayoría de las veces es una pérdida de tiempo, pues los alumnos no le toman el peso, contestan cualquier cosa y a veces la dejan en blanco" (E8:P14). En continuidad, un número reducido de profesores interculturales y tradicionales emplea la evaluación de proceso, lo cual se visualiza en la siguiente apreciación: "Considero que la evaluación formativa es importante, porque es una instancia para que los niños aprendan" (E31:P5). Por otra parte, un gran número de docentes emplea la evaluación sumativa, lo cual se manifiesta en los siguientes discursos; al respecto, un profesor tradicional expresa: "Buenos días, estudiantes guarden sus cuadernos porque hoy tienen prueba de la unidad que hemos visto, me imagino que estudiaron para la prueba..." (R9/OB7:P5). Asimismo, un docente intercultural declara: "La evaluación sumativa, la aplico una vez que paso todos los contenidos de una unidad, y ahí veo cuánto han aprendido" (E19:P23). En este sentido, las prácticas evaluativas están centradas en los resultados y no en la continuidad del proceso de enseñanza y aprendizaje. En complementación, la evaluación sumativa "permite comprobar el grado en que el alumno alcanzó los objetivos previstos y constatar la consecución de objetivos" (Blázquez Entonado \& Lucero Fustes, p. 250).
Código 5. Dominio del conocimiento disciplinar y didáctico

El código se refiere al dominio del conocimiento disciplinar y didáctico que poseen los profesores tradicionales e interculturales en sus prácticas de enseñanza.

En este contexto, se evidencia un desconocimiento de los conocimientos culturales mapuches tanto disciplinares como metodológicos de parte de los profesores tradicionales, lo cual se pone de manifiesto en el siguiente relato de un profesor intercultural: "Lo otro que veo es el desconocimiento de parte de los profesores tradicionales, respecto de los saberes culturales mapuche, ya que no tienen un manejo de los contenidos de la asignatura (lengua indígena) y de la metodología para enseñarlos" (E25:P33). Lo anterior denota un desconocimiento del contenido disciplinar; tampoco tienen el conocimiento didáctico del contenido para movilizar adecuadamente los procesos de enseñanza-aprendizaje en contextos interculturales. El interés por el CDC se debe, sobre todo, a que implica un conjunto de saberes que permite al profesor trasladar a la enseñanza el contenido de un determinado tópico (Acevedo Díaz, 2009).

\section{Código 6. Articulación pedagógica}

Este código da cuenta de si existe o no una articulación pedagógica entre profesores interculturales y profesores tradicionales, y su correspondiente argumentación.

Al respecto, no se evidencia articulación pedagógica, pues es posible visualizar que, en su mayoría, los profesores diseñan la enseñanza de manera individual, además de otras actividades. Lo anterior se manifiesta en la siguiente declaración: "La elaboración de las planificaciones la hacemos individualmente, puesto que no hay espacios para realizarla en forma colaborativa con los profesores interculturales y tradicionales que permitan intercambiar conocimientos y experiencias, para mejorar nuestras prácticas" (E12:P13). Un tercer profesor narra: "Nosotros generalmente planificamos individualmente, esto porque no existe una coordinación de parte del Jefe de Unidad Técnico Pedagógica [en adelante, JUTP] para la conformación de equipos de trabajo para el diseño de planificaciones, evaluaciones, guías, etc." (E17:P26). En este sentido, no existe una gestión de parte del JUTP que permita generar espacios para la reflexión pedagógica, a fin de que los profesores diseñen la enseñanza de forma colaborativa y constructiva. Al respecto, Segundo Quintriqueo, Héctor Torres, Maritza Gutiérrez y Dykssa Sáez (2011) proponen una metodológica intercultural sobre la base de una transposición didáctica en la cual las lógicas de conocimiento tanto occidental como indígena se plasmen en la planificación de la acción didáctica, con base en objetivos, recursos y estrategias didácticas. 
Código 7. Práctica profesional reflexiva

En este código se analizan las prácticas de los profesores y de los JUTP desde la práctica profesional reflexiva.

Se evidencian elementos que obstaculizan la práctica profesional reflexiva de los docentes interculturales y tradicionales. Al respecto, un profesor intercultural manifiesta: "No se generan los espacios para llevar a cabo la planificación en equipo y reflexionar sobre nuestras prácticas pedagógicas" (E19:P7). Del mismo modo, un profesor tradicional declara: "El JUTP entra a la clase, se sienta y luego después de un tiempo sale, pero nunca nos retroalimenta y ni reflexiona respecto de nuestras prácticas de enseñanza. Entonces uno queda en el aire, ya que solo se dedica a controlar" (E22:P34). De las evidencias expuestas, los profesores no reflexionan respecto de sus prácticas pedagógicas, al tiempo que se advierte una ausencia de retroalimentación y reflexión de parte de los JUTP. Esto implica que la práctica pedagógica de este directivo debiera orientarse hacia la reflexión permanente sobre la práctica del profesor (Schön, 1992).

\section{Código 8. Prejuicios}

Este código pone en evidencia los prejuicios que poseen los profesores tradicionales respecto de la cultura mapuche.

En este escenario, se observan prejuicios de parte de ciertos profesores tradicionales respecto de la lengua mapuche y los saberes culturales mapuche. Respecto de lo expuesto un profesor intercultural expresa: "En la educación básica intercultural, el factor que obstaculiza es el prejuicio hacia la cultura mapuche que tienen algunos profesores tradicionales respecto de los saberes culturales mapuche" (E22:P35). Una profesora declara: "A los niños no les sirve mucho el mapudungun (lengua mapuche) al momento de trabajar, ya que la mayoría por no decir todos, hablan español e inglés" (E23:P37). Al respecto, se observa que el mapudungun y los conocimientos culturales mapuches no son valorados por una minoría de los profesores tradicionales. En contraste con lo mencionado anteriormente, es de suma importancia reconocer el mapudungun, puesto que es una lengua con todas sus potencialidades $y$, por tanto, portadora de grandes conocimientos, emociones y sentimientos, que sin ella se perderían (Lara, 2012).

\section{Análisis de datos}

El diseño de la enseñanza está enmarcado en un modelo rígido con escasos espacios durante el desarrollo de la clase para reflexionar sobre los aprendizajes y sobre la práctica pedagógica. Por tanto, es posible indicar que el modelo pedagógico dominante está centrado en el docente. En contraste, Lee Shulman (2005) plantea que "la docencia se inicia cuando se 'reflexiona' en qué es lo que debe ser aprendido y cómo debe ser aprendido por los estudiantes". En esta lógica, el profesor debe hacer un análisis crítico y reflexivo sobre su práctica pedagógica con el objeto de mejorarla.

La metodología de enseñanza se focaliza en la clase frontal, puesto que el modelo pedagógico dominante reside en el docente y no en el alumno; por tanto, se enmarca en clases verticales, poco reflexivas. En tal sentido, las estrategias no estimulan el autoaprendizaje pues las prácticas están centradas en el profesor y no en el estudiante como constructor de su propio aprendizaje. Lo anterior obstaculiza los procesos de enseñanza-aprendizaje en contextos interculturales. Por tanto, se deben priorizar estrategias que lleven al aprender haciendo y aprender reflexionando (Quintriqueo, Torres, Gutiérrez \& Sáez, 2011).

En este contexto, los profesores tradicionales utilizan con menor frecuencia estrategias centradas en el aprendizaje, como la narración de cuentos, leyendas, lectura de textos, trabajo en grupo, que facilitan y estimulan el desarrollo de aprendizajes contextualizados. En sintonía con lo antepuesto, para Javier González García (2007), el objetivo común y fundamental de la narración de cuentos en el aula es avivar la imaginación y la creatividad del niño mediante una actividad lúdica que procura momentos de distensión y de recreo, y el desarrollo de la facilidad de comprensión y de expresión en el niño. Además, permite robustecer la comprensión del alumno de diversos temas que se le pueden ir presentando a lo largo de su formación y de su vida. Respecto del trabajo en grupo, Francisco Salvador Mata y José Luis Gallego Ortega (2012) plantean que:

Permite aprender a trabajar y a resolver tareas en grupo, cuyos miembros tienen diversos niveles de capacidad y de dominio de la tarea. En el grupo, todos los miembros comparten el liderazgo; el profesor debe estructurar la tarea y asignar una función a cada miembro del grupo (p. 362).

No obstante, la escasez y la baja frecuencia con que se emplean estas estrategias dificultan el desarrollo de aprendizajes de calidad. Por otra parte, algunos profesores interculturales declaran emplear estrategias didácticas contextualizadas tanto mapuches como occidentales pero con una menor frecuencia, como los conocimientos previos, el nütram (relato oral), ngülam (consejo), epew (cuentos), feyentun (leyendas), piam (mitos), pewmas (sueños) y el trabajo en grupo. La transmisión de conocimiento en el pueblo mapuche está basada en la "oralidad"; en este plano, Hilda Llanquinao 
Trabol (2009, p. 222) señala que "la educación mapuche tradicionalmente se desarrolla en el plano de la oralidad, que es la comunicación verbal que se da en un contexto determinado, sistema por el cual los mapuches han generado formas eficaces de transmitir su visión de mundo". Esta formación se realiza mediante el nütram. Según Víctor Toledo Llancaqueo (2005, p. 421), "es un relato oral transmitido de generación en generación mapuche". Asimismo, la enseñanza mapuche o transmisión de saberes está basada en el ngülam, que es el consejo, el cual se manifiesta de diversas maneras, puede ser por medio del epew (cuentos o relatos), feyentun (leyendas) y pewmas (sueños) (Consejo Nacional de la Cultura y las Artes, CNCA, 2011).

En este contexto, surge el gülhamtun (aconsejar), el cual se define como "un modo de organizar y transmitir el conocimiento a las nuevas generaciones" (Quintriqueo, 2010, p. 54). Esta forma de educación — mediante la "palabra" - busca potenciar habilidades propias de cada niño y descubrir sus cualidades personales. La transmisión de experiencias y conocimientos es fundamental en la formación de la persona, por consiguiente, esta metodología de enseñanza facilita la movilización de aprendizajes contextualizados y, por tanto, significativos. En consecuencia, según Quintriqueo (2010), es preciso que profesores tradicionales e interculturales repliquen estrategias metodológicas con un enfoque intercultural, basadas en la transposición didáctica, la cual permite transformar el contenido científico en contenido de enseñanza (Chevallard, 1991). En este sentido, lo contextualiza, temporaliza y personaliza en el ámbito que lo rodea (Silva, 2002).

Los docentes interculturales y tradicionales obtienen el conocimiento base para el desarrollo de la enseñanza de diversas fuentes: preparación profesional adquirida en su formación universitaria, información recabada en congresos y en la publicación de investigaciones. La investigación educativa tiene como propósito explorar detallada y minuciosamente un problema de conocimiento, así como exponer y publicar los descubrimientos que arroja la indagación. El cuidado que ponen los investigadores en su trabajo hace posible que el lector cuente con información confiable y comprenda cómo son los actores, las instituciones y las prácticas que se desarrollan en los espacios educativos. Así, los lectores de estos documentos podrán apoyarse en los resultados para profundizar en esos problemas de conocimiento, o para adentrarse en una problemática vinculada (Piña Osorio, 2013). Además, se evidencia un uso excesivo del texto escolar y esto dificultaría el aprendizaje; según Salvador Mata y Gallego Ortega (2012), los textos son aquellos que pueden aproximar la realidad al estudiante, a través de símbolos o imágenes. No obstante, Salvador Mata y Gallego Ortega señalan que "la visita a una fábrica de la localidad o de la zona en la que se sitúa la escuela puede ser un estímulo más motivador que estudiar en un libro de texto las características de la empresa" (p. 192). Además, solo los profesores interculturales consultan expertos. En efecto, "(...) el conocimiento se ubica como una dimensión humana, considerada como un repertorio de experiencias culturales, acumuladas en la memoria individual y colectiva a través del tiempo, y expresada en los discursos orales o escritos" (Quintriqueo \& Torres, 2013), según sea el tipo de cultura. En este contexto, los profesores interculturales recurren a la consulta a expertos, es el caso de los kimches: personas (sabios portadores del conocimiento social y cultural) para formar a niños y jóvenes en el contexto de la familia y comunidad (Quilaqueo Rapimán \& Quintriqueo, 2010). Los kimches poseen un conocimiento confiable, pues son personas sabias y portadores del conocimiento social y cultural mapuche sustentado en sus experiencias, 
lo cual facilita la construcción de aprendizajes contextualizados. Cabe mencionar que lo referido a lo "pedagógico y a un currículo contextualizado" no es advertido por ambos tipos de profesores.

En cuanto a las prácticas evaluativas, la mayoría de los docentes no valora la evaluación diagnóstica, asimismo, se observa que la minoría de profesores aplica la evaluación de proceso, pues desconocen su relevancia en la mejora de los procesos de enseñanza y aprendizaje. En tal sentido, un gran número de docentes tradicionales e interculturales centra sus prácticas en la evaluación sumativa, es decir, en los resultados. Según Carmen Ruiz Bueno (2001), la evaluación sumativa se aplica al producto, a los procesos terminados. Este tipo de evaluación está centrada en los resultados, y en la calificación, y no en el aprendizaje continuo, permanente de los estudiantes; además, carece de un aspecto retroalimentador. Respecto a lo anterior, Pedro Ahumada Acevedo (2001) plantea que "hoy más que nunca la evaluación debe constituir un proceso más que un suceso y, por tanto, interesa obtener evidencias centradas en el proceso de aprender más que en los resultados o productos (p. 24).

Además, se evidencia un desconocimiento de los saberes culturales mapuches tanto de la disciplina como de los métodos educativos de parte de los profesores tradicionales. Al respecto, Daniel Quilaqueo Rapimán y Segundo Quintriqueo (2010) expresan que hay un desconocimiento de los saberes educativos mapuches en la escuela. Al respecto, en el modelo de Shulman, además del conocimiento de la materia y del conocimiento general pedagógico, los profesores deben desarrollar un conocimiento específico: cómo enseñar su materia específica (Bolívar, 2005). En razón de esto, el docente debe tener un dominio tanto del conocimiento disciplinar como del conocimiento didáctico del contenido mapuche y occidental a fin de generar aprendizajes contextualizados y por consiguiente, de calidad.

Por otro lado, se evidencia la inexistencia de articulación pedagógica entre profesores tradicionales e interculturales, debido a que el JUTP no realiza una coordinación que permita generar espacios de reflexión pedagógica para diseñar la enseñanza de forma colaborativa y constructiva. En este contexto, María Verónica Maturana Cortés (2011) evidencia que los espacios de reflexión pedagógica son necesarios para tomar decisiones e imprescindibles para el trabajo docente y el logro de objetivos institucionales. En vínculo con lo expuesto, Segundo Quintriqueo (2010) expresa que debe haber un diálogo entre profesores y el JUTP para mejorar las prácticas pedagógicas.

En las prácticas pedagógicas de los profesores interculturales y tradicionales se evidencia la ausencia de reflexión y retroalimentación de estas. En razón de lo expuesto, Adad José Cassís Larraín (2011) plantea que:

La práctica profesional reflexiva permite al docente la construcción de conocimientos a través de la solución de problemas que se encuentran en la práctica; esto conlleva la construcción de un tipo de conocimiento desde las acciones para tomar decisiones mediante la utilización de estrategias y metodologías para innovar (p. 54).

Asimismo, los JUTP deben retroalimentar las prácticas de enseñanza de los docentes, a fin de buscar soluciones a los problemas que emergen durante su acción. De acuerdo con María Guadalupe Zoila Flores Orozco y María Soledad Ramírez Montoya (2009), la retroalimentación es una estrategia para la mejora educativa, pues es un elemento indispensable en el proceso de aprendizaje. En tal sentido, el profesor debe 
reflexionar sobre sus prácticas, al tiempo que el JUTP debe generar los espacios para estas y actuar como un profesional reflexivo y retroalimentador y, no como un controlador.

Finalmente, emergen prejuicios de parte de algunos profesores tradicionales respecto de los conocimientos mapuches y del mapudungun (lengua mapuche), que no son valorados. La resistencia de sus pares, los prejuicios y estereotipos hacia el conocimiento mapuche son aspectos que inciden en que se mantengan las estructuras curriculares que continúan con la reproducción de modelos educativos monoculturales (Quintriqueo \& McGinity Travers, 2009). Estos prejuicios eclipsan el pensamiento de los profesores tradicionales y por tanto, dificultan la articulación pedagógica contextualizada entre estos y los profesores interculturales.

\section{Conclusión}

A partir de la discusión de resultados, en relación con el "diseño de la enseñanza", es posible constatar un diseño rígido de manera que no responde a un modelo global e integrado, pues se visualizan pocos espacios para reflexionar sobre los aprendizajes. Por lo tanto, el modelo pedagógico dominante está centrado en el docente, con una orientación positivista, lo que obstaculiza el proceso de enseñanza y aprendizaje. Respecto a esto, Mario de Miguel Díaz (2005) plantea que la enseñanza debería estar centrada en el alumno y las capacidades de este, por tanto, la planificación debería sufrir un cambio en esta dirección.

En relación con la "metodología de enseñanza", la estrategia didáctica más utilizada en el proceso de enseñanza en el aula por profesores tradicionales e interculturales, se enmarca en un modelo de enseñanza vertical, que promueve actos didácticos poco reflexivos en el quehacer pedagógico. Por otra parte, algunos profesores interculturales utilizan estrategias propias de la cultura mapuche lo que facilita el traspaso del conocimiento contextualizado. Esto se da a través de la oralidad, es decir, a través del nütram (discurso oral), ngülam (consejo), epew (cuentos), feyentun (leyendas), piam (mitos), pewmas (sueños). Asimismo, emplean conocimientos previos y el trabajo en grupo. Por otro lado, los docentes tradicionales emplean los cuentos, leyendas, trabajo en grupo, la lectura de textos. A pesar de que se emplean con menor frecuencia, las estrategias mapuches son esenciales, pues facilitan la vehiculización de los procesos didácticos, asimismo, son necesarias para generar aprendizajes contextualizados y de calidad, y por consiguiente óptimas para la formación de estudiantes mapuches y no mapuches.

En lo que respecta a "fuentes de conocimiento", los profesores interculturales y tradicionales recurren a la preparación profesional adquirida en su formación universitaria y de la información recabada en congresos y publicaciones de investigaciones, lo cual facilita el diseño de la enseñanza. Asimismo, hacen uso excesivo del texto escolar, lo que dificulta los aprendizajes contextualizados de los estudiantes. Por otra parte, solo los profesores interculturales recurren a la experiencia de expertos (kimches), que poseen conocimientos de la cultura mapuche, lo cual contribuye a la contextualización de la enseñanza y por tanto, de los aprendizajes. Desde las "prácticas evaluativas", se evidencia que la evaluación diagnóstica no es suficientemente valorada. Asimismo, una minoría de los profesores aplica la evaluación de proceso, pues dan cuenta de su relevancia en el progreso de los aprendizajes. Por otra parte, un gran número de profesores interculturales y tradicionales utiliza la evaluación sumativa, la cual está centrada en los resultados, y no en el aprendizaje continuo y permanente 
de los estudiantes. Además, carece de un carácter continuo y retroalimentador, lo cual obstaculiza la mejora de los aprendizajes. En cuanto al código "dominio del conocimiento disciplinario y didáctico" es posible constatar de parte de los profesores tradicionales un desconocimiento de los contenidos y de las formas de enseñanza de la cultura mapuche. Lo anterior dificulta la enseñanza situada y por tanto, la generación de aprendizajes de calidad.

Respecto a la "articulación pedagógica", esta no se advierte; por lo tanto, es posible concluir que el diseño de la enseñanza tiene un carácter individualista, pues no se visualiza una articulación entre profesores. Esto se debe a la falta de una gestión de parte del JUTP que permita generar espacios para la articulación y reflexión en el diseño de la enseñanza desde un enfoque colaborativo y constructivo. En este contexto, emerge "la práctica profesional reflexiva", en la que se evidencia que entre profesores tradicionales e interculturales no reflexionan sobre sus prácticas, ni en conjunto con el JUTP; además, este no las retroalimenta, lo que resulta preocupante, pues el profesor actuará de manera mecanizada, irreflexivamente. Asimismo, su práctica seguirá sujeta de acuerdo con sus concepciones y creencias, las cuales condicionan la toma de decisiones.

Finalmente, es posible constatar que un número reducido de profesores tradicionales no valora los saberes culturales mapuche, ni la lengua mapuche, lo cual dificulta la articulación pedagógica entre profesores tradicionales e interculturales y, además, el desarrollo de una adecuada práctica pedagógica contextualizada. Asimismo, Daniel Quilaqueo Rapimán (2006) plantea que podrían superarse los prejuicios hacia los saberes mapuche, si se consideraran los conocimientos tradicionales de la memoria social mapuche transmitidos como saberes educativos y al mismo tiempo, los conocimientos del medio escolar. Al respecto, los profesores tradicionales deben superar los prejuicios a fin de avanzar en la formación y educación de calidad de estudiantes mapuches y no mapuches.

Las conclusiones de esta investigación resultan preocupantes, pues ponen en evidencia que la educación escolar precisa de una relación educativa con el saber científico y el conocimiento cultural mapuche para la formación de niños y adolescentes, es decir, se debe romper con la forma tradicional de enseñanza homogeneizadora. Esto implica para el profesorado realizar procesos de enseñanza pertinentes al contexto, que permitan generar aprendizajes culturalmente significativos, para niños mapuche y no mapuche. Ello requiere de innovaciones en las prácticas pedagógicas de los profesores que enseñan en contextos educativos interculturales. Esto, con la finalidad de superar en parte la hegemonía de una educación centrada en la cultura occidental y, por tanto, entregar una formación pertinente e integral.

En concordancia con lo expuesto en el párrafo anterior, es fundamental estudiar los procesos de transposición didáctica que realizan los profesores interculturales y tradicionales que actúan en contextos de diversidad cultural a fin de buscar mecanismos, estrategias y medios didácticos para movilizar de forma eficiente los procesos de enseñanza-aprendizaje. Esto permitiría, por un lado, comprender el desarrollo de los procesos didácticos y, por otro, avanzar en el conocimiento de la enseñanza que se lleva a cabo en contextos de diversidad cultural. En suma, cabe señalar que, desde el punto de vista epistemológico, las prácticas de enseñanza que se desarrollan en contextos interculturales ayudan a comprender y establecer rutas pedagógicas que permitan potenciar los procesos pedagógicos de los docentes que actúan en este tipo de contextos, esto con el fin de superar los problemas que históricamente ha generado la monoculturalidad que impera en la escuela para alumnos de origen tanto mapuche como no mapuche (Quilaqueo, Quintriqueo \& San Martín, 2011). Por tanto, se invita a profesores tradicionales e interculturales a profundizar y optimizar los procesos de transposición didáctica en contextos interculturales, desde una práctica de enseñanza contextualizada, reflexiva y crítica a fin de mejorar en parte la calidad de la educación de los estudiantes situados en contextos interculturales.

El estudio tiene como principal limitación el muestreo, el cual puede ser ampliado para así considerar la propia heterogeneidad de la sociedad mapuche y de los saberes construidos a partir de la relación que ha existido entre el sistema escolar chileno y los saberes transmitidos desde las comunidades indígenas.

Se recomienda realizar investigaciones en métodos educativos mapuche que contribuyan al desarrollo de la enseñanza en un sentido integral e inclusivo en las asignaturas de lengua indígena, lenguaje y comunicación, matemáticas, historia, geografía y ciencias sociales, y ciencias naturales. Esto, con la finalidad de contextualizar el diseño del currículo en lo referido a la enseñanza en contextos educativos interculturales, para mejorar la calidad de los aprendizajes y la convivencia de estudiantes mapuche y no mapuche.

Además, se sugiere investigar sobre el papel que desempeña la escuela en la articulación de saberes y conocimientos mapuches con los conocimientos escolares convencionales. Esto permitiría favorecer las relaciones dialógicas entre la cultura mapuche y occidental, a fin de facilitar la construcción de una sociedad más inclusiva, con una conciencia reflexiva y crítica. Asimismo, contribuiría al diseño de proyectos educativos en común entre ambas culturas. 


\section{Sobre los autores}

Juan Carlos Beltrán-Véliz es profesor de Educación Básica, Universidad Central de Chile. Magíster en Ciencias de la Educación, mención Currículum y Evaluación, Universidad Mayor de Temuco, Chile. Profesor guía de tesis de posgrado en la Facultad de Educación, Universidad Mayor de Temuco, Chile. Doctorante por la Universidad de la Frontera, Temuco, Chile.

Juan Guillermo Mansilla-Sepúlveda es decano de la Facultad de Educación, Universidad Católica de Temuco, Chile. Profesor de Estado en Historia, Geografía y Educación Cívica. Licenciado en Educación. Magíster en Desarrollo Regional y Local, Universidad Humanismo Cristiano, Chile. Doctor en Filosofía, Universidad Pontificia de Salamanca, España. Profesor de la Facultad de Educación, Universidad Católica de Temuco, Chile.

Carlos Baldemir Del Valle-Rojas es decano de la Facultad de Educación, Ciencias Sociales y Humanidades, Universidad de la Frontera, Chile. Periodista, Licenciado y Magíster en Comunicación por la Universidad de la Frontera, Temuco, Chile. Doctor en Comunicación, Universidad de Sevilla, España. Posdoctorado en el programa avanzado de Cultura Contemporánea, Universidad Federal de Río de Janeiro, Brasil.

Braulio Ademir Navarro-Aburto es doctor en Ciencias de la Educación. Magíster en Motricidad infantil, Universidad Mayor, Chile. Docente del Departamento de Educación Física, Deportes y Recreación de la Universidad de la Frontera, Temuco, Chile.

\section{Referencias}

Acevedo Díaz, J. A. (2009). Conocimiento didáctico del contenido para la enseñanza de la naturaleza de la ciencia. Revista Eureka sobre Enseñanza y Divulgación de las Ciencias, 6 (1), 21-46. Disponible en: http://www. redalyc.org/pdf/920/92012998003.pdf

Aguado, M. (1991). La educación intercultural: conceptos, paradigmas, realizaciones. Madrid: Dykinson.

Ahumada Acevedo, P. (2001). La evaluación en una concepción de aprendizaje significativo. Valparaíso: Salesianos S. A.

Bastiani Gómez, J.; Ruiz-Montoya, L.; Estrada Lugo, E.; Cruz Salazar, T. \& Aparicio Quintanilla, J. A. (2012). Práctica docente, castellanización, burocracia y centralización de la educación como limitaciones del éxito pedagógico en la región Ch'ol, Chiapas. Perfiles Educativos, 34 (135), 8-25. Disponible en: http://www.redalyc.org/articulo.oa?id=13223042002

Beltrán Véliz, J. C. (2014). Factores que dificultan la gestión pedagógica curricular de los jefes de Unidades Técnico Pedagógicas. Revista Mexicana de Investigación Educativa, 19 (62), 939-961. Disponible en: http://www. redalyc.org/pdf/140/14031461014.pdf

Beltrán Véliz, J. \& Osses-Bustingorry, S. (2018). Transposición didáctica de saberes culturales mapuche en escuelas situadas en contextos interculturales. Revista Latinoamericana de Ciencias Sociales, Niñez y Juventud, 16 (2), 669-684. doi:https://doi.org/10.11600/1692715x.16202. Disponible en: http://revistaumanizales.cinde.org.co/rlcsnj/index.php/Revista-Lati noamericana/article/view/2039/944

Berry, A.; Loughran, J. \& Driel, J. H. van (2008). Editorial. Revisiting the Roots of Pedagogical Content Knowledge. International Journal of Science Education, 30 (10), 1271-1279.

Blázquez Entonado, F. \& Lucero Fustes, M. (2012). La evaluación en educación. En A. Medina Rivilla \& F. Salvador Mata. Didáctica general, 243-270. $2^{\text {a }}$ ed., Madrid: Pearson Educación. Disponible en: http://ceum-morelos. edu.mx/libros/didacticageneral.pdf 
Bolívar, A. (2005). Conocimiento didáctico del contenido y didácticas específicas. Profesorado, Revista de Currículum y Formación del Profesorado, 9 (2), 1-39. Disponible en: https://www.ugr.es/ recfpro/rev92ART6.pdf

Cassís Larraín, A. J. (2011). Donald Schön: una práctica profesional reflexiva en la universidad. Revista Compás Empresarial, 3 (5), 54-58. Disponible en: http://www.sep.pue.gob.mx/component/k2/item/download/ 389 40920d6e9a2d19a671eac6734b77ff67

Chevallard, Y. (1991). La transposición didáctica: del saber sabio al saber enseñado. Buenos Aires: Aique Grupo Editor S.A.

Clermont, C. P.; Borko, H. \& Krajcik, J. S. (1994). Comparative Study of the Pedagogical Content Knowledge of Experienced and Novice Chemical Demonstrators. Journal of Research in Science Teaching, 31 (4), 419 441. https://doi.org/10.1002/tea.3660310409

Cochran-Smith, M. \& Fries, K. (2005). The AERA Panel on Research and Teacher Education: Context and Goals. En M. Cochran-Smith \& K. Zeichner (eds.). Studying Teacher Education. The Report of the AERA [American Educational Research Association] Panel on Research and Teacher Education, 37-68. Mahwah, New Jersey: Lawrence Erlbaum Associates.

Consejo Nacional de la Cultura y las Artes, CNCA (2011). Diagnóstico del desarrollo cultural del pueblo mapuche. Región de la Araucanía. Disponible en: http://www.cultura.gob.cl/wp-content/uploads/2013/04/ Estudio-Diagnostico-del-Desarrollo-Cultural-del-Pueblo-Mapuche.pdf

Díaz-Aguado, M. J. (2002). Educación intercultural y aprendizaje cooperativo. Madrid: Pirámide.

Fennema, E. \& Franke, M. (1992). Teacher's Knowledge and Its Impact. En D. A. Grouws (ed.). Handbook of Research on Mathematics Teaching and Learning: A Project of the National Council of Teachers of the Mathematics, 147-164. New York: MacMillan Publishing Co.

Flick, U. (2007). Introducción a la investigación cualitativa. Madrid: Morata.

Flores, G. \& Ramírez, M. (2009). Interrelación de la evaluación de los aprendizajes con la retroalimentación como estrategia para la mejora educativa. En Memorias del XVIII Encuentro Internacional de Educación a Distancia. Universidad de Guadalajara. Disponible en: http://www. ruv.itesm.mx/convenio/catedra/recursos/material/ci_22.pdf

Garritz, A. (2006). Historia y retos de la formación de profesores (Algo más sobre Lee Shulman). Educación Química, 17 (3), 322-326. Disponible en: www.educacionquimica.info/include/downloadfile. php?pdf=pdf933.pdf

Garritz, A. \& Trinidad-Velasco, R. (2004). El conocimiento pedagógico del contenido. Educación Química, 15 (2), 98-103. Disponible en: https:// andoni.garritz.com/documentos/edit_cpc.pdf

Gess-Newsome, J. (1999). Pedagogical Content Knowledge: An Introduction and Orientation. En J. Gess-Newsome \& N. G. Lederman (eds.). Examining Pedagogical Content Knowledge: The Construct and its Implications for Science Education, 3-17. Dordrecht, The Netherlands: Kluwer Academic Publishers.

Giroux, H. (1997). Los profesores como intelectuales. Barcelona: Paidós.

González García, J. (2007). Las narraciones y la tradición oral en el aula de preescolar. SUMMA Psicológica UST, 4 (2), 129-136.

Grisales-Franco, M. \& González-Agudelo, E. (2009). El saber sabio y el saber enseñado: un problema para la didáctica universitaria. Educación y Educadores, 12 (2), 77-86. Disponible en: http://educacionyeduca dores.unisabana.edu.co/index.php/eye/article/view/1486/1655

Gurdián-Fernández, A. (2007). El paradigma cualitativo en la investigación socio-educativa. San José, Costa Rica: Coordinación Educativa y 
Cultural Centroamericana (CECC), Agencia Española de Cooperación Internacional (AECI). Disponible en: https://web.ua.es/en/ice/documentos/ recursos/materiales/el-paradigma-cualitativoen-la-investigacion-socio-educativa.pdf

Jordán, J. A. (1999). El profesorado ante la educación intercultural. En M. À. Essomba Gelabert \& E. Barandica i Pairet (coords.). Construir la escuela intercultural. Reflexiones y propuestas para trabajar la diversidad étnica y cultural, 65-74. Barcelona: Graó.

Kuhn, T. (1962). La estructura de las revoluciones científicas. Ciudad de México: Fondo de Cultura Económica.

Lara-Millapan, M. (2012). Aprender a leer y escribir en lengua mapudungun, como elemento de recuperación y promoción de la cultura mapuche en la sociedad del siglo XXI. (Tesis doctoral). Universidad Autónoma de Barcelona, España. Disponible en: https://www.tdx.cat/bitstream/handle/ 10803/107892/milm1de1. pdf?sequence=1

Llanquinao-Trabol, H. (2009). Valores de la educación tradicional mapuche: posibles contribuciones al sistema educativo chileno. (Tesis doctoral inédita). Universidad de Barcelona, Barcelona, España. Disponible en: https://www.tdx.cat/bits tream/handle/10803/2356/HLLT_TESIS.pdf

Mansilla-Sepúlveda, J. \& Beltrán-Véliz, J. (2013). Coherencia entre las estrategias didácticas y las creencias curriculares de los docentes de segundo ciclo, a partir de las actividades didácticas. Perfiles Educativos, 35 (139), 25-39. Disponible en: http:// www.redalyc.org/articulo.oa?id=13225611012

Marcelo-García, C. (2001). El aprendizaje de los formadores en tiempos de cambio. La aportación de las redes y el caso de la Red Andaluza de Profesionales de la Formación. Profesorado. Revista de Currículum y Formación de Profesorado, 5 (1), 29-44. http://www.ugr.es/ recfpro/rev51ART2.pdf

Maturana-Cortés, M. V. (2011). Espacios de reflexión pedagógica, una aproximación de los profesores en liceos municipales y particular subvencionados de la comuna de Vallenar. (Tesis de magíster inédita). Universidad de Chile. Santiago, Chile. Disponible en: http://repositorio.uchile.cl/ tesis/uchile/2011/cs-maturana_m/pdfAmont/csmaturana_m.pdf

Mellado-Hernández, M. \& Chaucono-Catrinao, J. (2016). Liderazgo pedagógico para reestructurar creencias docentes y mejorar prácticas de aula en contexto mapuche. Revista Electrónica Educare, 20 (1), 1-18. doi.org/10.15359/ree.201.18. Disponible en: http://www.redalyc.org/ pdf/1941/194143011018.pdf

Miguel-Díaz, M. de (2005). Cambio de paradigma metodológico en la Educación Superior. Exigencias que conlleva. Cuadernos de Integración Europea, 2, 16-27. Disponible en: http://cdeuv.es/images/ documents/2005-CIE-02.pdf.pdf

Mulholland, J. \& Wallace, J. (2005). Growing the Tree of Teacher Knowledge: Ten Years of Learning to Teach Elementary Science. Journal of Research in Science Teaching, 42 (7), 767-790.

Muñoz-Justicia, J. (2005). Análisis cualitativo de datos textuales con ATLAS ti. Universidad Autónoma de Barcelona. Disponible en: http://psicologiaso cial.uab.es/juan/index.php/docs-mainmenu-89/ category/12-mis-textos?download $=2$ : analisisde-datos-textuales-con-atlas-ti-5

Organización para la Cooperación y el Desarrollo Económicos, OCDE (2005). Teachers Matter: Attracting, Developing and Retaining Effective Teachers. Paris: OCDE. Disponible en: http://www.oecd. org/education/school/attractingdevelopingan dretainingeffectiveteachers-finalreportteachers matter.htm

Pellón-Arcaya, M.; Mansilla-Sepúlveda, J. \& San Martín -Cantero, D. (2009). Desafíos para la transposición didáctica y conocimiento didáctico del contenido en docentes de anatomía: obstáculos y proyecciones. International Journal of Morphology, 27 (3), 743-750. Disponible en: https://scielo. conicyt.cl/pdf/ijmorphol/v27n3/art18.pdf

Pérez-Serrano, G. (2001). Investigación cualitativa. Retos e interrogantes. $3^{a}$ ed., Madrid: La Muralla.

Piña-Osorio, J. M. (2013). Investigación educativa ¿para qué? Perfiles Educativos, 35 (139), 3-6. Disponible en: http://www.redalyc.org/pdf/132/ 13225611010.pdf

Quidel-Catrilaf, G. (2011). Estrategias de enseñanza de la lengua mapunzugun en el marco del PEIB [Programa de Educación Intercultural Bilingüe] Mineduc-Orígenes. Cuadernos Interculturales, 9 (16), 61-80. Disponible en: http://www.redalyc. org/pdf/552/55218731005.pdf

Quilaqueo-Rapimán, D. (2006). Valores educativos mapuche para la formación de persona desde el discurso de kimches. Estudios Pedagógicos (Valdivia), 32 (2), 73-86. Disponible en: http://revis tas.uach.cl/pdf/estped/v32n2/art04.pdf

Quilaqueo-Rapimán, D. \& Quintriqueo, S. (2010). Saberes educativos mapuches: un análisis desde la perspectiva de los kimches. Polis, Revista de la Universidad Bolivariana, 9 (26), 337-360. Disponible en: https:// journals.openedition.org/polis/808\#text

Quilaqueo, D.; Quintriqueo, S. \& San Martín, D. (2011). Contenido de aprendizajes educativos mapuches para el marco conceptual de un currículum escolar intercultural. Estudios Pedagógicos (Valdivia), 37 (2), 233-248. Disponible en: https://scielo.co nicyt.cl/pdf/estped/v37n2/art14.pdf 
Quintriqueo, S. (2010). Implicancias de un modelo curricular monocultural en contexto mapuche. Santiago de Chile: Universidad Católica de Temuco. Disponible en: http://milenio.uct.cl/wp-content/ uploads/2014/08/4049i-Implicancias-de-unmodelo-curricular.indd_.pdf

Quintriqueo, S. \& McGinity Travers, M. (2009). Implicancias de un modelo monocultural curricular en la construcción de la identidad sociocultural de alumnos/as mapuches de la IX región de la Araucanía, Chile. Estudios Pedagógicos (Valdivia), 35 (2), 173-188. Disponible en: http://www.redalyc. org/articulo.oa? id=173514137010

Quintriqueo, S.; Morales, S.; Quilaqueo, D. \& Arias, K. (2016). Interculturalidad para la formación inicial docente: desafíos para construir un diálogo intercultural. Temuco, Chile: Ediciones Universidad Católica de Temuco.

Quintriqueo, S. \& Torres, H. (2013). Construcción de conocimiento mapuche y su relación con el conocimiento escolar. Estudios Pedagógicos (Valdivia), 39 (1), 199-216. Disponible en: https://scie lo.conicyt.cl/pdf/estped/v39n1/art12.pdf

Quintriqueo, S.; Torres, H.; Gutiérrez, M. \& Sáez, D. (2011). Articulación entre el conocimiento cultural mapuche y el conocimiento escolar en ciencia. Educación y Educadores, 14 (3), 475492. Disponible en: http://educacionyeduca dores.unisabana.edu.co/index.php/eye/article/ view/1934/2595

Rowland, T. \& Turner, F. (2007). Developing and Using the "Knowledge Quartet". A Framework for the Observation of Mathematics Teaching. The Mathematics Educator, 10 (1), 107-123.

Ruiz-Bueno, C. (2001). La evaluación de programas de formación de formadores en el contexto de la formación en y para la empresa (Tesis doctoral inédita). Universidad Autónoma de Barcelona. Barcelona, España. Disponible en: https://www. tdx.cat/handle/10803/5003

Ruiz-Olabuénaga, J. (2012). Metodología de la investigación cualitativa. $5^{\mathrm{a}}$ ed. Bilbao: Universidad de Deusto.

Sáez-Alonso, R. (2006). La educación intercultural. Revista de Educación, 339, 859-881. Disponible en: http://www.revistaeducacion.mec.es/re339/ re339a37.pdf

Salvador-Mata, F. \& Gallego-Ortega, J. L. (2012). Enfoque didáctico para la socialización. En A. Medina Rivilla \& F. Salvador Mata. Didáctica general, 352-386. Madrid: Pearson Educación. Disponible en: http://ceum-morelos.edu.mx/libros/dida cticageneral.pdf
Sartorello, S. C. (2014). La co-teorización intercultural de un modelo curricular en Chiapas, México. Revista Mexicana de Investigación Educativa, 19 (60), 73-101. Disponible en: http://www.redalyc. org/pdf/140/14029405005.pdf

Schön, D. (1992). La formación de profesionales reflexivos. Hacia un nuevo diseño de la enseñanza y el aprendizaje en las profesiones. Barcelona: Paidós.

Serrano Ruiz, J. (1998). El papel del maestro en la Educación Intercultural Bilingüe. Revista Iberoamericana de Educación, 17, 91-104. Disponible en: https://rieoei.org/historico/oeivirt/ rie17a04.htm

Shulman, L. (1999). Foreword. En J. Gess-Newsome \& N. G. Lederman (eds.). Examining Pedagogical Content Knowledge: the Construct and its Implications for Science Teaching, ix-xii. Dordrecht, The Netherlands: Kluwer Academic Publishers.

Shulman, L. (2004). The Wisdom of Practice: Essays on Teaching, Learning, and Learning to Teach. San Francisco: Jossey-Bass.

Shulman, L. (2005). Conocimiento y enseñanza: fundamentos de la nueva reforma. Profesorado. Revista de Currículum y Formación de Profesorado, 9 (2), 1-30. Disponible en: https://www.ugr. es/ recfpro/rev92ART1.pdf

Silva, É. E. (2002). La Transposición Didáctica (LTD) herramienta para la acción en la Zona de Desarrollo Próximo (ZDP). Omnia, 8 (1-2), 1-18. Disponible en: http://produccioncientificaluz.org/index. php/omnia/article/view/7051/7040

Strauss, A. \& Corbin, J. (2002). Bases de la investigación cualitativa. Técnicas y procedimientos para desarrollar la teoría fundamentada. Medellín, Colombia: Universidad de Antioquia.

Toledo Llancaqueo, V. (2005). La memoria de las tierras antiguas tocando las puertas al derecho. Políticas de la memoria mapuche en la transición chilena. En F. Gómez. El derecho a la memoria, 421-497. Araba Kalea, País Vasco: Itxaropena.

Walsh, C. (2009). Interculturalidad crítica y educación intercultural. Disponible en: http://www.uchile. $\mathrm{cl} /$ documentos/interculturalidad-critica-y-edu cacion-intercultural_110597_0_2405.pdf

Wertsch, J. (1991). Voces de la mente. Un enfoque sociocultural para el estudio de la acción mediada. Madrid: Visor.

Zamorano, A. (2015). La práctica de la enseñanza de las matemáticas a través de las situaciones de contingencia. (Tesis doctoral). Universidad Autónoma de Barcelona. Barcelona, España. Disponible en: https://www.tesisenred.net/bitstream/hand le/10803/288225/azv1de1.pdf?sequence $=1$ 